\title{
Clinical features of ovarian large-cell neuroendocrine carcinoma: Four case reports and review of the literature
}

\author{
TAKAFUMI OSHITA ${ }^{1}$, TOMOMI YAMAZAKI ${ }^{2}$, YUMIKO AKIMOTO ${ }^{2}$, HIROTOSHI TANIMOTO ${ }^{2}$, \\ NOBUTAKA NAGAI ${ }^{2}$, MASARU MITAO $^{2}$, AKIO SAKATANI $^{3}$ and MAYUMI KANEKO ${ }^{3}$ \\ ${ }^{1}$ Department of Obstetrics and Gynecology, Miyoshi Central Hospital, Miyoshi, Hiroshima; \\ Departments of ${ }^{2}$ Obstetrics and Gynecology, and ${ }^{3}$ Pathology, Hiroshima City Asa Hospital, Hiroshima, Japan
}

Received June 17, 2011; Accepted July 27, 2011

DOI: $10.3892 / \mathrm{etm} .2011 .325$

\begin{abstract}
The objective of the present study was to present 4 recently encountered ovarian large-cell neuroendocrine carcinoma (LCNEC) cases, and to evaluate their clinicopathological features in the context of the previously reported 29 LCNEC cases. First, we described the clinical features of 4 recently encountered cases. Routine H\&E staining and immunohistochemistry for CD56, synaptophysin and chromogranin A were performed on sections of both the LCNEC and epithelial carcinoma components. Clinical data for the total of 33 LCNEC cases were summarized, and the Kaplan-Meier survival curve was estimated. Our cases were observed in women aged 42-81 years. One case is clinically classified as FIGO stage IV with multiple metastases, and the others are classified as FIGO stages Ic, IIc and IIIb by post-surgical findings. Pathological features, assessed by H\&E staining, were similar to lung LCNEC, and at least one neuroendocrine marker was positive staining in both LCNEC and the epithelial component. One case was pure type LCNEC and the others were mixed carcinoma. Paclitaxel/carboplatin chemotherapy was performed for all cases and 3 of the 4 treatments were effective. The prognoses of our cases were as follows: 1 in stage Ic died from the disease after only 2 months, but the others survived, with or without recurrence, for 32-64 months, whereas the total 5-year survival of the 33 LCNEC cases was $34.9 \%$. In summary, our 3 LCNEC cases revealed ordinary chemo-sensitivity, resulting in a better prognosis than those previously described, apart from 1 case which exhibited aggressive behavior. For the future, a retrospective survey to elucidate the prognostic factors and prospective clinical studies to evaluate the efficacy of treatment modalities of ovarian LCNEC are necessary, particularly for aggressive LCNEC cases.
\end{abstract}

Correspondence to: Dr Takafumi Oshita, Department of Obstetrics and Gynecology, Miyoshi Central Hospital, 531 HigashiSakeya, Miyoshi, Hiroshima, Japan

E-mail: toshita@hicat.ne.jp

Key words: ovarian cancer, large-cell neuroendocrine carcinoma, immunohistochemistry, case report, review

\section{Introduction}

The World Health Organization (WHO) defines large-cell neuroendocrine carcinoma (LCNEC) as a tumor with a large nucleus and a tendency to have neuroendocrine differentiation, and it defines ovarian LCNECs as miscellaneous tumors (1). In Japan, the term LCNEC was described for the first time in The General Rules for Clinical and Pathological Management of Ovarian Tumors by the Japan Society of Obstetrics and Gynecology and the Japanese Society of Pathology in December 2009. Until these classifications were established, LCNEC had been classified as anaplastic or poorly differentiated carcinoma and was synonymous with undifferentiated carcinoma of the non-small cell neuroendocrine type $(1,2)$. According to the WHO classification of lung cancer (3), the pathological structures by H\&E staining reveal a characteristic organization, such as round nest and sheet-like formation with frequently central coagulative necrosis, and rosette-like formation of tumor cells is often observed everywhere. The nucleus is large with granular or coarse chromatin, a prominent nucleolus and moderate or abundant cytoplasm. Additionally, neuroendocrine differentiation by immunohistochemical analysis, such as chromogranin A, synaptophysin and CD56, is required to confirm the diagnosis of LCNEC. This cancer is also generally accompanied by surface epithelial-stromal tumors, and the LCNEC components metastasize relatively early, resulting in poor prognosis (1). To date, only 29 cases of ovarian LCNEC have been reported (4-17). Thus, we describe our 4 recently encountered cases and evaluate the clinicopathological features of ovarian LCNEC from 33 primary cases, including previously reported cases.

\section{Materials and methods}

Initially, we described the clinical courses of our 4 cases. Then, for all 33 available LCNEC cases, we summarized the clinicopathological findings, such as patient age, FIGO stage, tumor marker values, operation and intra-abdominal findings, chemotherapy regimens and outcomes (4-17). The Kaplan-Meier overall survival rate was estimated by analyzing the survival time data reported in the literature with STAT view software for Windows. Routine H\&E staining and immunohistochemical staining for CD56, synaptophysin and 
chromogranin A as neuroendocrine markers were performed in sections of both LCNEC and epithelial carcinoma components. The antibody clones, dilutions and sources that we used are as follows: CD56 (1B6, 1/1; Nichirei), chromogranin A (DAK-A3, 1/100; Dako) and synaptophysin (SY38, 1/100; Dako).

\section{Case reports}

Case 1. A 66-year-old, gravida 2, parity 2, post-menopausal woman was found to have multiple lung nodules in a chest $\mathrm{X}$-ray obtained during a routine medical examination. She was referred to us because of her pelvic mass. The patient also had a metastatic tumor in her vagina, which was diagnosed as undifferentiated carcinoma based on histological examination. A computed tomography (CT) examination revealed that the pelvic tumor was composed of cystic and solid components, suggesting malignancy. Her CA125 level was elevated to $6,595 \mathrm{U} / \mathrm{ml}$; therefore, we clinically defined this case as FIGO (International Federation of Gynecology and Obstetrics) clinical stage IV ovarian cancer. Consequently, we initiated paclitaxel/carboplatin (TC) chemotherapy in the neoadjuvant setting. Clinical partial response (PR), including complete remission of multiple lung disease, was acquired by RECIST criteria after 4 cycles of chemotherapy; therefore, interval-debulking surgery was performed, including bilateral salpingo-oophorectomy, hysterectomy, omentectomy and peritoneal biopsy. There were no malignant findings, except for the existing viable cancer cells in the right ovary. Although adjuvant and maintenance chemotherapy were continued, brain metastasis was observed 17 months after the start of initial treatment. Neurosurgeons resected the tumor and the brain tumor pathology was similar to not only the primary vaginal tumor, but also to ordinary lung LCNEC. Immunohistochemical analyses for neuroendocrine markers were performed, and positive staining for chromogranin A was acquired. As a result, the brain tumor was diagnosed as metastatic LCNEC that had originated from the ovary, despite lacking an epithelial component. The patient received wholebrain radiation therapy, and during the 64 months post initial treatment she did not experience tumor recurrence.

Case 2. An 80 -year-old, gravida 2, parity 2, post-menopausal woman was found to have an abdominal mass during a routine medical examination. The fist-sized, cystic tumor was palpable near the left side of the uterus. Ultrasonography, $\mathrm{CT}$ and magnetic resonance imaging (MRI) examinations revealed a $7-\mathrm{cm}$ left ovarian tumor with solid and cystic components, and hydrosalpinx. Her CA125 level was relatively high at $204.3 \mathrm{U} / \mathrm{ml}$. Her Pap cervical cancer screening was normal. As part of her complete surgical treatment, she underwent the following: bilateral salpingo-oophorectomy, hysterectomy, pelvic lymphadenectomy, omentectomy and appendectomy, which macroscopically resulted in no residual tumor. Para-aortic lymph nodes were not swollen upon palpation. Histopathologically, this tumor was diagnosed as LCNEC with an endometrioid adenocarcinoma component. The tumor had already ruptured and invaded into the left fallopian tube and the parametrium with positive peritoneal cytology, and it was classified as FIGO surgical stage IIc ovarian cancer (pT2cN0M0). Six courses of postoperative TC [175 mg/m $/ \mathrm{m}^{2}$ paclitaxel and carboplatin (CBDCA) at AUC 6.0] chemotherapy were carried out and during the 40 months post initial treatment there were no recurrent signs.

Case 3. A 65-year-old, nulligravida, post-menopausal woman complained of nausea and abdominal distension with continuous pain. She was referred to our hospital because of an abdominal mass. CT and MRI examinations revealed an $11-\mathrm{cm}$ cystic abdominal ovarian tumor with enhanced nodule formation. Her CA125 and CA19-9 levels were 77.0 and $776.5 \mathrm{U} / \mathrm{ml}$, respectively. Her Pap smear of the uterine cervix and endometrium was negative. Owing to her continuous abdominal pain, we performed an emergency operation, including bilateral salpingo-oophorectomy, hysterectomy and omentectomy; macroscopically, there was no residual tumor. Pelvic and para-aortic lymph nodes were not swollen upon palpation. The tumor had spontaneously ruptured, which was suggested as the cause of her abdominal pain. Peritoneal washing cytology was negative. On pathological examination, most of the tumor had a characteristic sheet or nest formation with central and peripheral coagulative necrosis, and the diagnosis of LCNEC was confirmed by CD56-positive immunohistochemistry. The remaining tumor was diagnosed as endometrioid adenocarcinoma with squamous differentiation. Although we planned postoperative adjuvant chemotherapy for this FIGO surgical stage Ic ovarian cancer (pT1cNxM0), the patient was morbid with nausea, vomiting and pain as a result of her abdominal distension within 2 weeks of the operation. CT examination revealed an occlusive ileus due to the $10-\mathrm{cm}$ recurrent pelvic mass, and liver metastasis and regional lymphadenopathy had already appeared. The patient died 2 months after the surgery and exhibited no response to the TC chemotherapy.

Case 4. A 42-year-old, gravida 3, parity 3 woman with a regular menstrual cycle was discovered to have a lower abdominal mass at a local clinic; she was then referred to our hospital. CT and MRI examinations revealed an ovarian tumor composed of cystic and solid parts. Her CA125 and STN levels were elevated to 775.2 and $139.3 \mathrm{U} / \mathrm{ml}$, respectively. Pap smears of the uterine cervix and endometrium were both negative. The tumor disseminated to the abdominal cavity, particularly the omentum and the Douglas peritoneum; as a result, optimal debulking surgery was performed, including bilateral salpingooophorectomy, hysterectomy with peritoneum resection of the Douglas pouch, omentectomy and retroperitoneal lymphadenectomy. Histopathological diagnosis was LCNEC, which contained a component of endometrioid adenocarcinoma with weakly positive staining for CD56 and chromogranin A. All disseminated tumors in the right ovary, parametrium, uterus, Douglas peritoneum and omentum were histologically diagnosed as LCNEC. The patient received 6 cycles of postoperative TC (175 mg/m² paclitaxel and CBDCA at AUC 6.0) chemotherapy for FIGO surgical stage IIIb ovarian cancer (pT3bNOM0), and during the 32 months after initial therapy she was alive with no signs of recurrence.

Pathological findings. Macroscopic findings on the cut surface of the tumors were not specific compared to the usual type of ovarian cancer. The solid component was slightly elastic with 
A

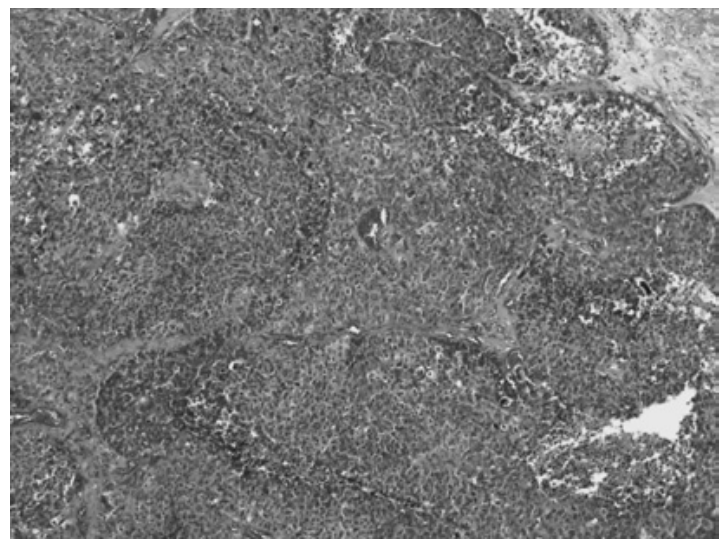

C

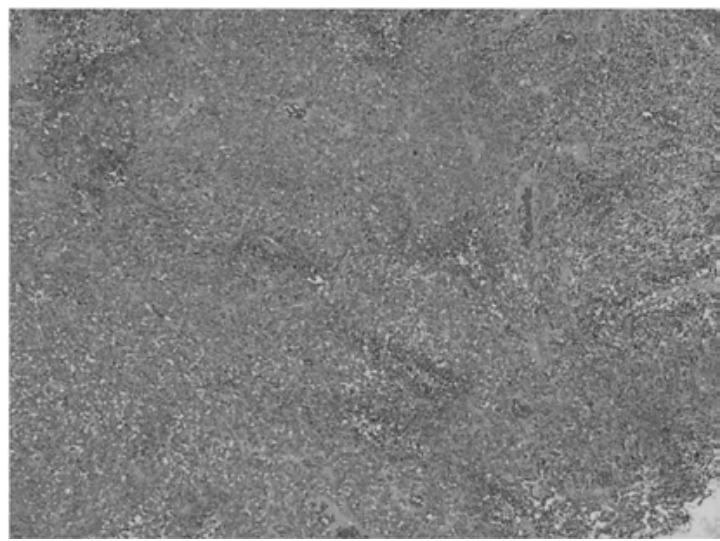

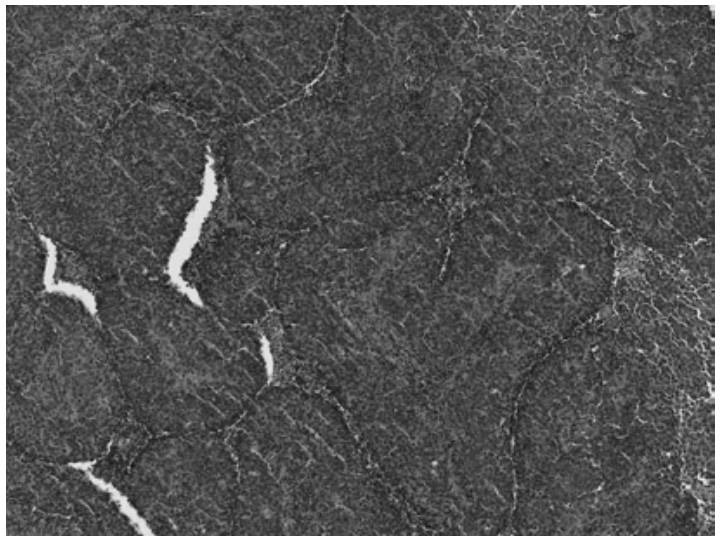

D

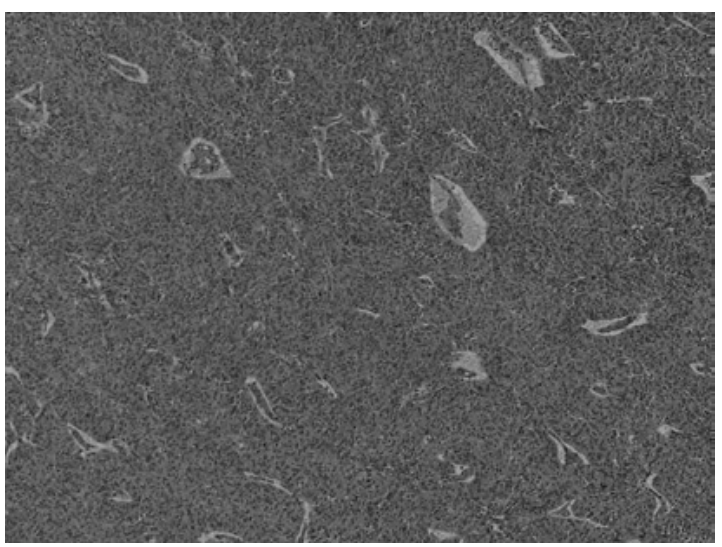

Figure 1. Microscopic findings (x40, H\&E staining). (A) Case 1; (B) Case 2; (C) Case 3; (D) Case 4. Nest formation with central coagulative necrosis and surrounding hemorrhage.

A

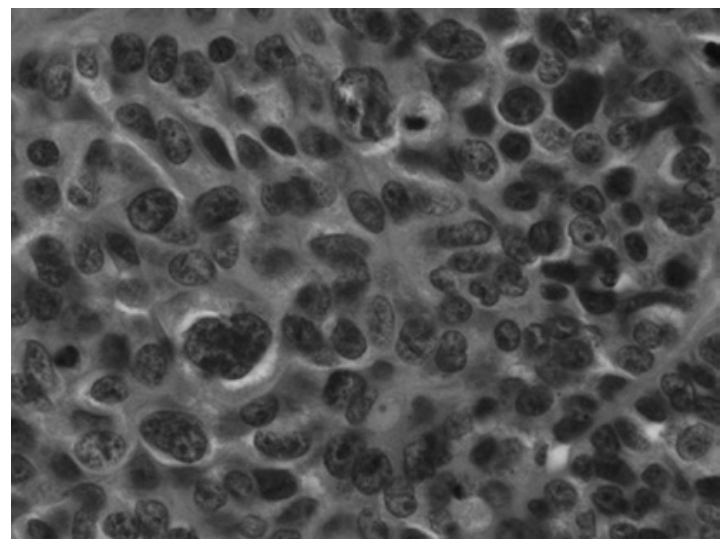

B

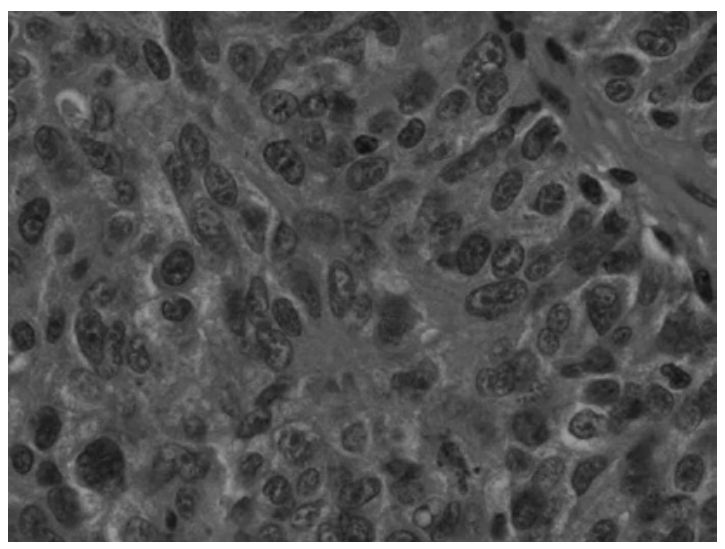

Figure 2. Microscopic findings (x400, H\&E staining). (A) Case 2; (B) Case 4. Rosette formations in the nest. Tumor cells have round-to-oval nuclei with hyperchromatin and mitosis.

a light gray color and had hemorrhage and necrosis. All cases had characteristic findings on microscopic examination of the H\&E-stained slides (Fig. 1A-D). Sheet or nest formations were observed, which had central coagulative necrosis with scanty stroma or massive hemorrhage surrounding the nest. Tumor cells were arranged in a palisading pattern at the periphery of the nests, and frequently showed rosette-like formation (Fig. 2A and B). The tumor cells had large round-to-oval nuclei, sometimes with prominent nucleoli, granular or coarse chromatin and relatively abundant basophilic cytoplasm. In particular, the nuclear polymorphism in Case 3 was very strong with high mitotic activity. In addition, adenocarcinoma components were observed adjacent to the LCNEC component. For example, Case 2 showed well differentiated endometrioid adenocarcinoma, Case 3 had poorly differentiated endometrioid adenocarcinoma with squamous differentiation and mucinous adenocarcinoma, and Case 4 had poorly differentiated endometrioid adenocarcinoma. At the time of diagnosis, these H\&E findings led us to initially doubt that the tumors were LCNECs; however, we finally defined the tumors more decisively based on positive immunohistochemical results for neuroendocrine markers, such as CD56, chromogranin A and synaptophysin. In the LCNEC component, 3 of 4 cases $(75 \%)$ were partially or diffusely positive for CD56, 3 of 4 cases $(75 \%)$ 
A

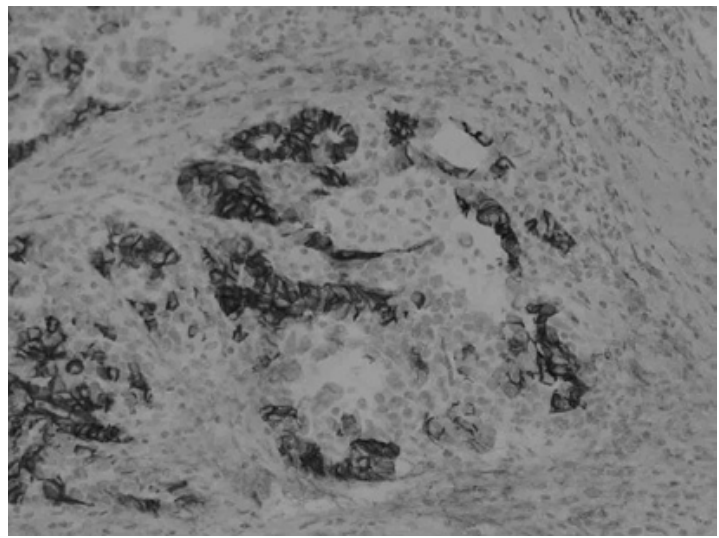

B

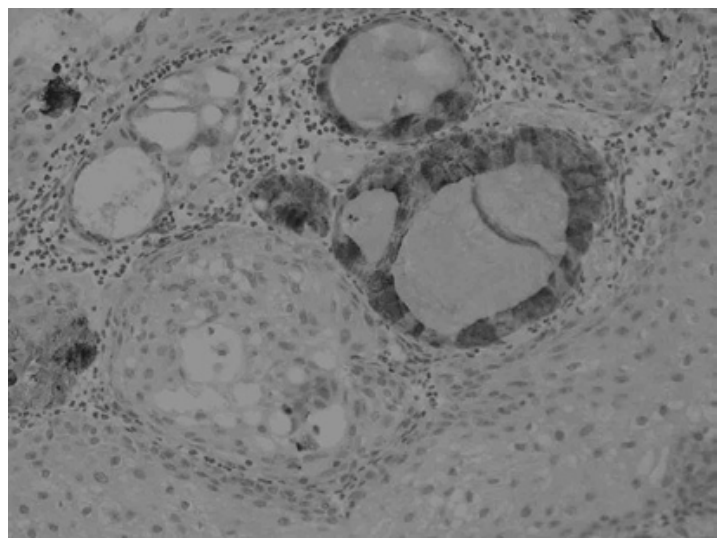

Figure 3. Microscopic findings (x100, immunohistochemical staining of the carcinoma component). (A) Positive immunohistochemical staining for CD56 in Case 2; (B) positive immunohistochemical staining for chromogranin A in Case 3.

were partially positive for chromogranin A, and all of the cases were negative for synaptophysin (Fig. 3A and B). On the other hand, in the adjacent carcinoma component, a similar staining pattern was observed compared to the LCNEC component.

Clinicopathological features of LCNEC - literature review. The clinicopathological summary of the 33 LCNEC cases is presented in Table I (4-17). The median age of the patients was 55 years (range 22-81). The FIGO surgical stages were: 15 stage I, 3 stage II, 8 stage III, 6 stage IV and 1 unknown stage. There was no laterality, and the median tumor diameter was $14 \mathrm{~cm}$ (range 5-30). The CA125 level was elevated in 11 cases, including our 4 cases, similar to what is usually found in ovarian cancer $(4,6,9,10,14,16,17)$. The epithelial components in 29 cases of ovarian LCNEC, excluding the 4 pure type LCNEC cases, were as follows: 17 cases (56.7\%) were mucinous tumors (benign, borderline malignancy and malignancy), 8 cases (26.7\%) were endometrioid adenocarcinomas, 3 cases $(10 \%)$ were mature cystic teratomas, 2 cases (6.7\%) were adenocarcinomas, not otherwise specified, 2 cases $(6.7 \%)$ were serous adenocarcinomas and 1 case $(3.3 \%)$ was a benign ovarian cyst (4-14). In the 21 cases described for which the patients had undergone surgical treatment, $16(76.2 \%)$ cases had complete surgery, 4 (19.0\%) had optimal surgery and $1(4.8 \%)$ had suboptimal surgery. Platinum-based chemotherapy was performed in most of the cases. The most popular recurrence sites were both pelvis \pm abdomen and liver, found in 5 out of the 11 cases $(45.4 \%)$ described in the literature. The
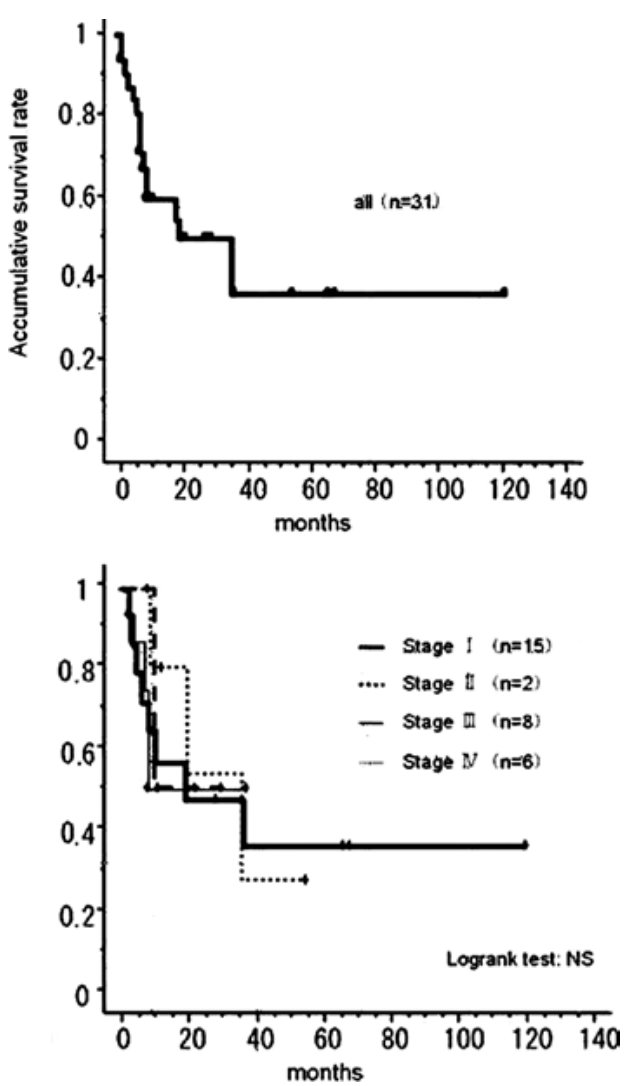

Figure 4. Overall survival of LCNEC (Kaplan-Meier method). The total 5-year survival was $34.9 \%$.

next most popular sites were as follows: lymph nodes (4 out of 11 cases, 36.4\%), brain ( 3 out of 11 cases, 27.2\%) and bone ( 2 out of 11 cases, 18.2\%). A Kaplan-Meier survival curve of the 33 cases is shown in Fig. 4. Twelve patients died within 12 months, such that the total 5-year survival was $34.9 \%$, a value that was $35.3 \%$ even in stage I patients.

\section{Discussion}

Only 33 primary ovarian LCNEC cases have been reported in the literature, including our 4 cases. LCNEC is a relatively new classification, and gynecologic oncologists are still not familiar with its name. The most difficult differential diagnoses are thought to be poorly differentiated carcinoma and undifferentiated carcinoma. In diagnosing LCNEC, both the presence of an epithelial component and morphological neuroendocrine differentiation, such as rosette formation, are useful. In the confusing case of distinguishing LCNEC from poorly differentiated adenocarcinoma, it is first necessary to doubt in favor of LCNEC by characteristic H\&E findings, and then to confirm its diagnosis through positive immunohistochemical results for neuroendocrine markers (3). On the other hand, in a case that lacks an epithelial component, undifferentiated carcinoma is the most important differential diagnosis. Although LCNEC is synonymous with undifferentiated carcinoma of the non-small cell neuroendocrine type according to the WHO classification (1), we posit that finding morphologic neuroendocrine differentiation, such as rosette-like formation, requires classification as LCNEC. Of course, it is important that secondary ovarian LCNEC from the lung is excluded 


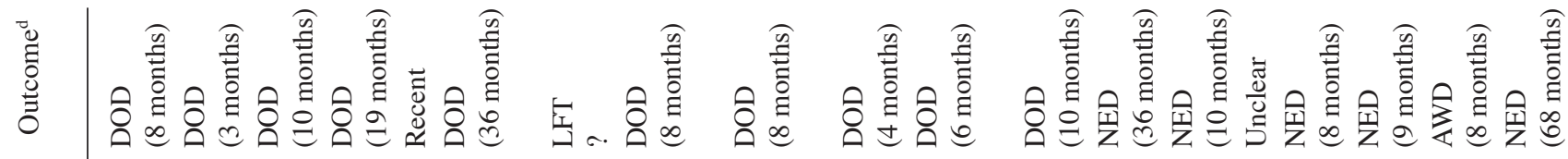

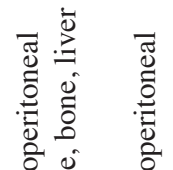

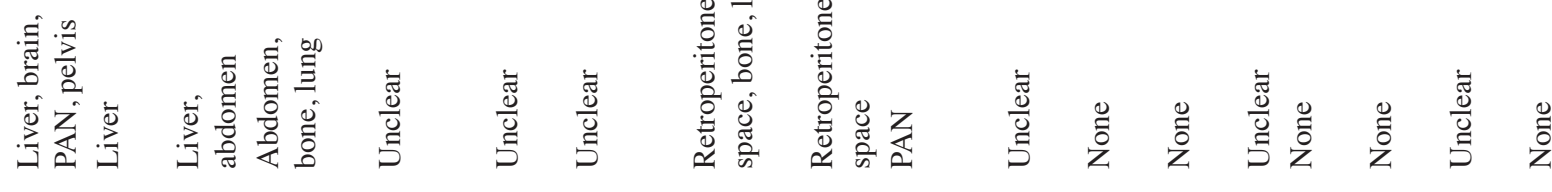

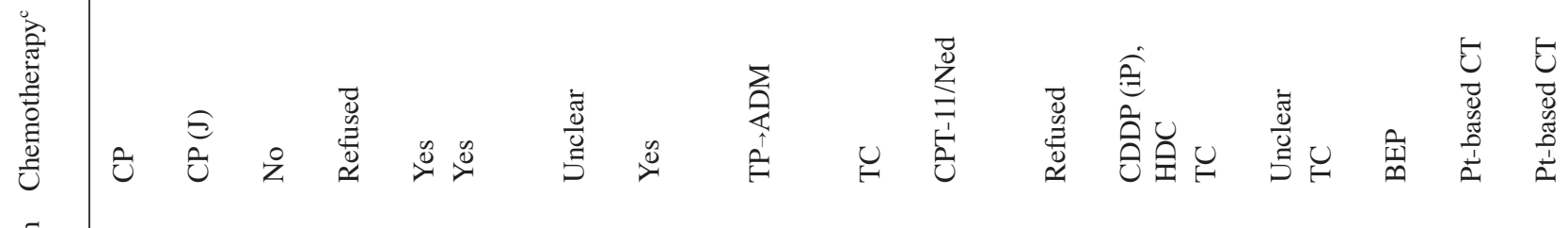

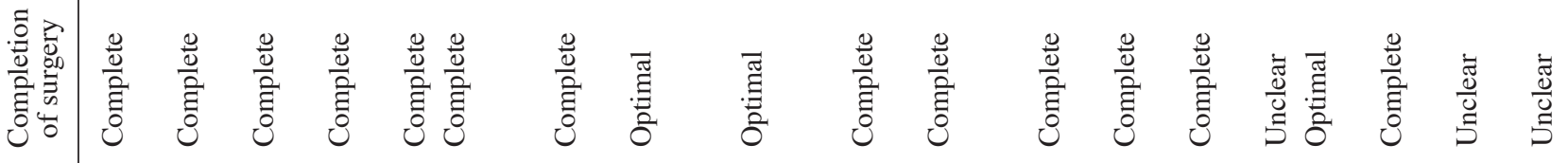

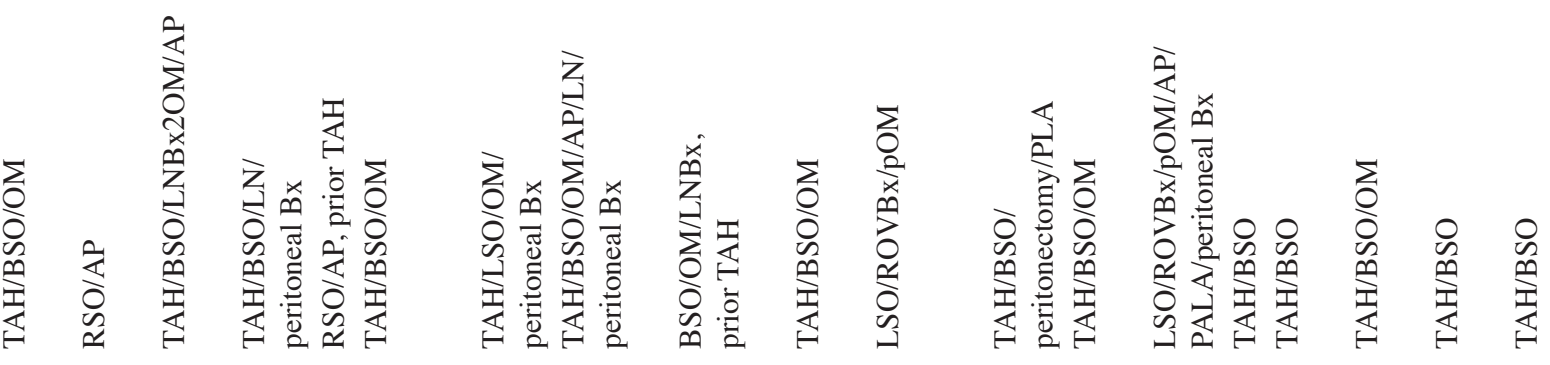

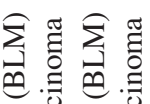

票苛

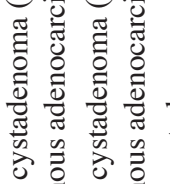

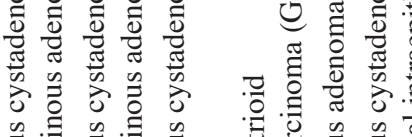

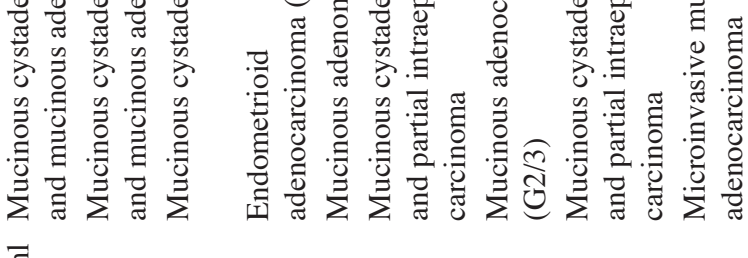

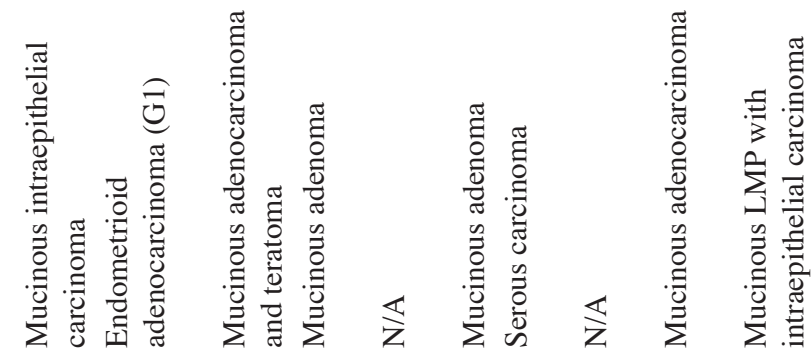

है
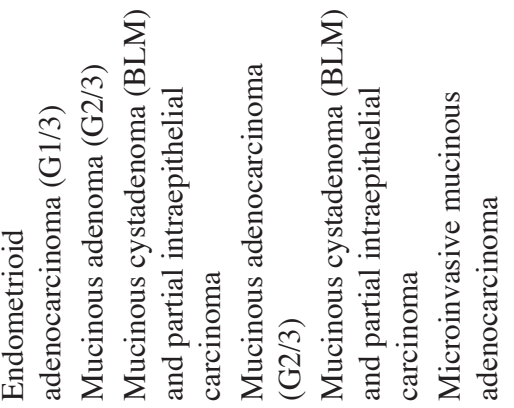

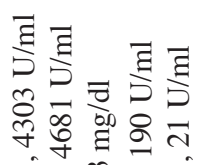

ह

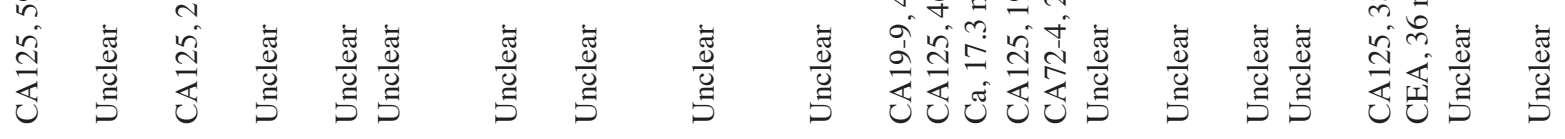

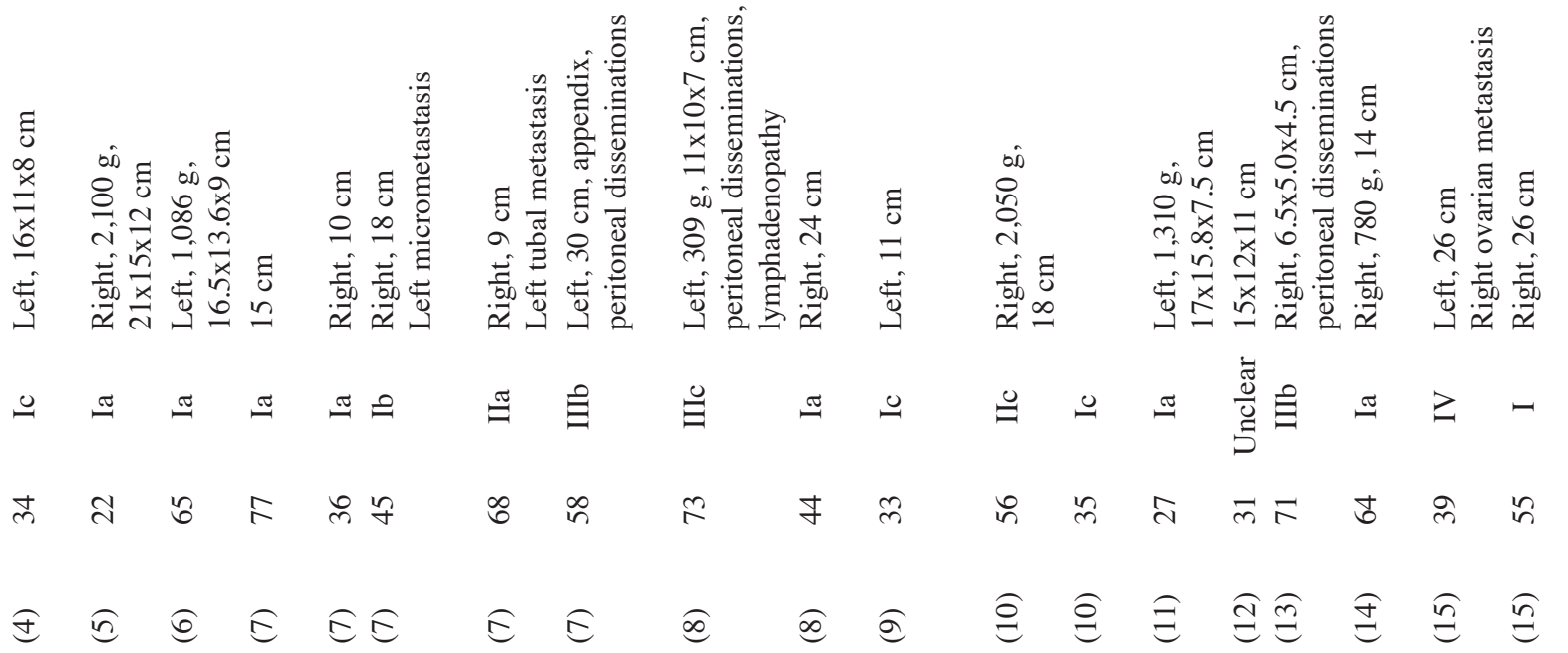




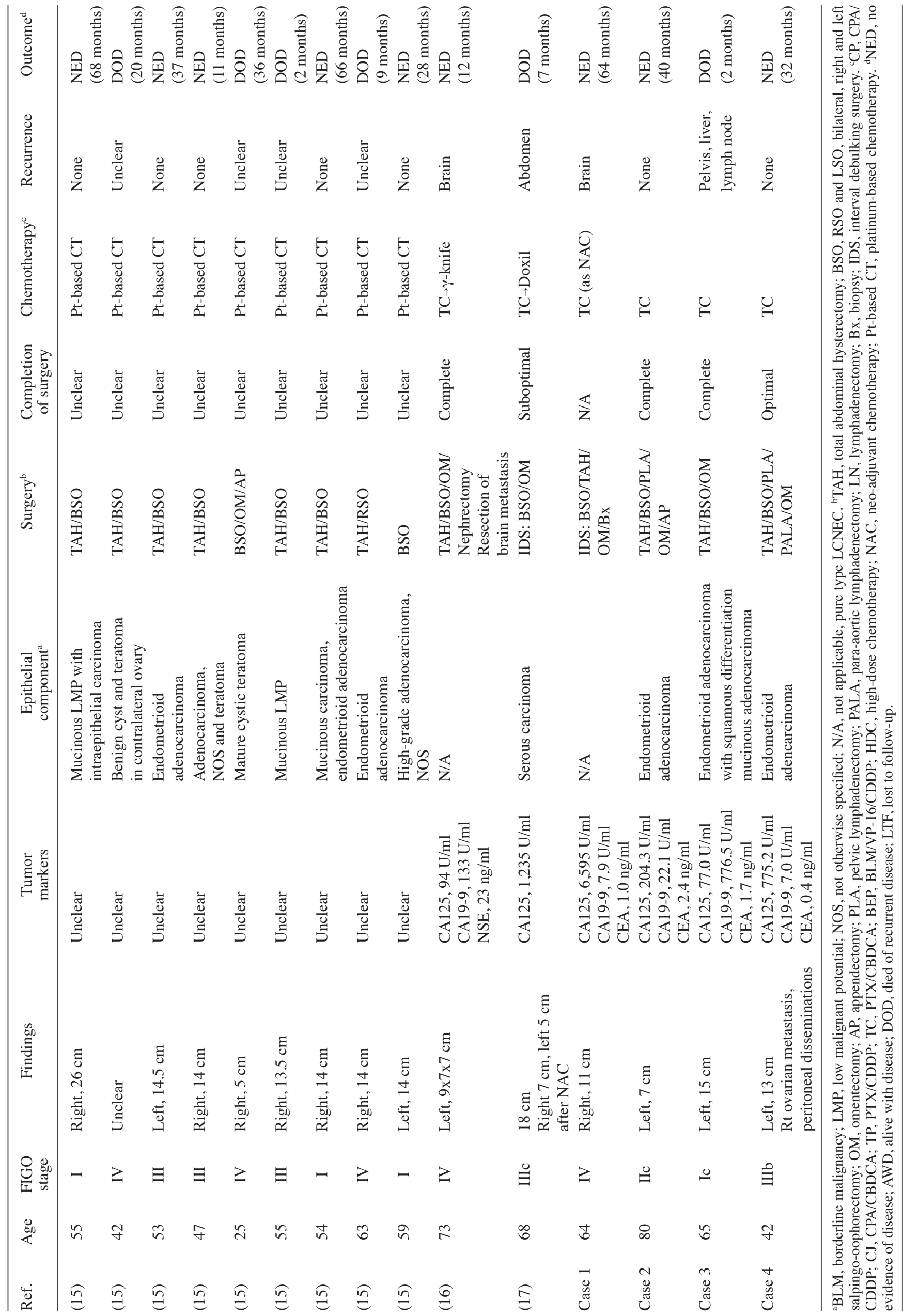


Table II. Immunohistochemical analysis of 33 LCNEC cases.

\begin{tabular}{|c|c|c|c|c|c|c|c|c|c|c|}
\hline \multirow[t]{3}{*}{ Ref. } & \multicolumn{5}{|c|}{ LCNEC component } & \multicolumn{5}{|c|}{ Epithelial component } \\
\hline & \multirow{2}{*}{$\frac{\begin{array}{c}\text { Epithelial } \\
\text { marker }^{\mathrm{a}}\end{array}}{\mathrm{CK}}$} & \multicolumn{4}{|c|}{ Neuroendocrine markers ${ }^{\mathrm{b}}$} & \multirow{2}{*}{$\frac{\begin{array}{c}\text { Epithelial } \\
\text { marker }^{\mathrm{a}}\end{array}}{\mathrm{CK}}$} & \multicolumn{4}{|c|}{ Neuroendocrine markers ${ }^{\mathrm{b}}$} \\
\hline & & CD56 & CG & SP & NSE & & CD56 & $\mathrm{CG}$ & SP & NSE \\
\hline (4) & + & N/A & + & N/A & \pm & + & N/A & + & N/A & N/A \\
\hline (5) & + & - & + & + & N/A & + & N/A & + & N/A & N/A \\
\hline (6) & + & N/A & + & + & \pm & N/A & N/A & N/A & N/A & N/A \\
\hline (7) & + & N/A & + & N/A & + & + & N/A & \pm & N/A & + \\
\hline (7) & + & N/A & + & N/A & + & + & N/A & + & N/A & \pm \\
\hline (7) & + & N/A & + & N/A & + & + & N/A & + & N/A & + \\
\hline (7) & + & N/A & + & N/A & + & + & N/A & + & N/A & + \\
\hline (7) & + & N/A & + & N/A & + & + & N/A & + & N/A & + \\
\hline (8) & + & N/A & + & + & N/A & + & N/A & + & - & N/A \\
\hline (8) & + & N/A & + & + & N/A & + & N/A & + & - & N/A \\
\hline (9) & N/A & N/A & + & + & + & N/A & N/A & \pm & - & - \\
\hline (10) & N/A & N/A & + & + & + & N/A & N/A & N/A & N/A & N/A \\
\hline (10) & N/A & N/A & + & \pm & + & N/A & N/A & N/A & N/A & $\mathrm{N} / \mathrm{A}$ \\
\hline (11) & + & N/A & + & N/A & N/A & & \multicolumn{4}{|c|}{ Absence of epithelial component } \\
\hline (12) & + & N/A & + & + & N/A & N/A & N/A & $\mathrm{N} / \mathrm{A}$ & N/A & $\mathrm{N} / \mathrm{A}$ \\
\hline (13) & \pm & + & + & + & N/A & + & - & - & - & N/A \\
\hline (14) & + & + & + & + & + & & \multicolumn{4}{|c|}{ Absence of epithelial component } \\
\hline$(15)$ & + & + & + & + & N/A & N/A & N/A & N/A & N/A & $\mathrm{N} / \mathrm{A}$ \\
\hline (15) & N/A & N/A & + & $\mathrm{N} / \mathrm{A}$ & $\mathrm{N} / \mathrm{A}$ & N/A & N/A & N/A & N/A & N/A \\
\hline (15) & + & + & + & + & N/A & N/A & N/A & N/A & N/A & N/A \\
\hline (15) & + & N/A & + & + & N/A & N/A & N/A & N/A & N/A & N/A \\
\hline (15) & + & + & - & + & N/A & N/A & N/A & N/A & N/A & N/A \\
\hline (15) & + & - & \pm & \pm & N/A & N/A & N/A & N/A & N/A & N/A \\
\hline (15) & \pm & N/A & - & N/A & + & N/A & N/A & N/A & N/A & N/A \\
\hline (15) & + & - & + & + & N/A & N/A & N/A & N/A & N/A & N/A \\
\hline (15) & + & - & - & \pm & N/A & N/A & N/A & N/A & N/A & N/A \\
\hline (15) & + & + & \pm & + & N/A & N/A & N/A & N/A & N/A & N/A \\
\hline (16) & + & + & + & + & N/A & & \multicolumn{4}{|c|}{ Absence of epithelial component } \\
\hline (17) & \pm & N/A & + & + & N/A & + & N/A & - & - & $\mathrm{N} / \mathrm{A}$ \\
\hline Case 1 & N/A & - & \pm & - & N/A & & \multicolumn{4}{|c|}{ Absence of epithelial component } \\
\hline Case 2 & N/A & + & - & - & $\mathrm{N} / \mathrm{A}$ & $\mathrm{N} / \mathrm{A}$ & + & - & - & $\mathrm{N} / \mathrm{A}$ \\
\hline Case 3 & N/A & + & \pm & - & N/A & N/A & + & \pm & + & N/A \\
\hline Case 4 & N/A & \pm & \pm & - & N/A & N/A & \pm & - & - & N/A \\
\hline
\end{tabular}

${ }^{\mathrm{a} C K}$, cytokeratin. ${ }^{\mathrm{b}} \mathrm{CD} 56, \mathrm{~N}-\mathrm{CAM}$; CG, chromogranin A; SP, synaptophysin; NSE, neuron-specific enolase; N/A, not applicable.

clinically. In Case 1, we made the clinical diagnosis of ovarian, rather than lung LCNEC because of both multiple nodules and the larger size of the ovarian tumor nodules.

Ovarian LCNEC is also characterized by the presence of surface epithelial-stromal tumors with benign, borderline or malignant behavior, and the presence of only LCNEC components at the metastatic site $(1,9)$. In fact, both metastatic brain tumors in Case 1 and the widely disseminated tumor to the abdominal cavity in Case 4 were diagnosed not as epithelial, but LCNEC components. Mucinous tumors and endometrioid adenocarcinoma were obviously predominant. However, in 2 cases with serous adenocarcinoma, both Draganova-
Tacheva et al (17) and Choi et al (13) concluded that each LCNEC component and serous adenocarcinoma component had different origins, meaning they arose independently, on the basis of the immunohistochemical pattern or microsatellite instability (MSI) analysis. Notably, neuroendocrine markers, such as chromogranin, synaptophysin and NSE, were detected in the epithelial component as well as in the LCNEC component in the same patient by immunohistochemistry $(4,5,7-9,17)$. These results are shown in Table II. This suggests that the LCNEC and epithelial components have similar biological characteristics, even though they differ in regards to morphology. 
The prognosis of ovarian LCNEC is recognized to be extremely poor (1); our survey also revealed that the total 5-year survival was $34.9 \%$, and still only $35.3 \%$ for stage I cases, even though in over $95 \%$ of these cases complete or optimal surgery was performed. There was such a high incidence in recurrence not only to the abdominal cavity, but also to specific sites that differed from the usual ovarian cancer distribution. This suggests that LCNEC may have strong lymph-vascular space invasion, which contributes to its poor prognosis. Case 3 showed extremely rapid progression with pelvic mass formation, liver metastasis and pelvic lymphadenopathy within only 2 weeks after primary surgery; moreover, TC chemotherapy was not effective in this patient. Similar cases have been previously reported $(4,5,8)$ and provide evidence that ovarian LCNEC is a malignant neoplasm with aggressive behavior.

However, we found that, except for the aforementioned aggressive case, our other cases were sensitive to TC chemotherapy and have to date demonstrated relatively good outcomes. Veras et al conducted a study at the MD Anderson Cancer Center and reported that 3 stage I cases of LCNEC acquired long-term survival for 22-68 months, and even stage III or IV cases revealed similar prognosis with standard surgery that was followed by adjuvant platinum-based chemotherapy (15). As a result, we suggest that most LCNEC cases are as chemotherapy-sensitive as common ovarian cancer. When these tumors are poorly responsive to TC chemotherapy, second-line chemotherapy regimens, such as cisplatin/vinorelbine, cisplatin/etoposide, cisplatin/vinblastine, cisplatin/gemcitabine and cisplatin/docetaxel, should be taken into consideration according to the National Comprehensive Cancer Network (NCCN) guidelines for primary lung LCNEC (18). Moreover, to our surprise, in 2 LCNEC cases that included brain metastasis, both tumor resection and adjuvant radiotherapy resulted in patient survival, to date (16). Thus, in cases of local recurrence, the combination of chemotherapy with aggressive surgery and adjuvant radiation therapy should be taken into consideration as a possible treatment strategy.

In summary, only 33 ovarian LCNEC cases have been reported to date. The term $\mathrm{LCNEC}$ has been unfamiliar to gynecologic oncologists not only in Japan, but also worldwide, and its criteria for diagnosis may be considered vague. As a result, certain LCNEC cases may have been inaccurately classified as undifferentiated or poorly differentiated adenocarcinoma. Generally, the prognosis of LCNEC has been recognized as extremely poor owing to its biologically aggressive behavior. However, we suggest that some ovarian LCNECs reveal more favorable prognosis than previously reported, particularly because of their ordinary chemo-sensitivity. A retrospective survey to elucidate the prognostic factors and prospective clinical studies to evaluate the efficacy of treatment modalities of ovarian LCNEC are necessary, particularly for aggressive LCNEC cases.

\section{References}

1. Tavassoli FA and Devilee P: World Health Organization Classification of Tumours. Pathology and Genetics of Tumours of the Breast and Female Genital Organs. IARC Press, Lyon, 2003.

2. Scully RE, Young RH and Clement PB: Atlas of Tumor Pathology. Tumors of the Ovary, Maldeveloped Gonads, Fallopian Tube, and Broad Ligament. Armed Forces Institute of Pathology. Washington, DC, 1996.

3. Travis WD, Brambilla E, Muller-Hermelink HK, et al: World Health Organization Classification of Tumours. Pathology and Genetics of Tumours of the Lung, Pleura, Thumus and Heart. IARC Press, Lyon, 2004.

4. Collins RJ, Cheung A, Ngan HY, et al: Primary mixed neuroendocrine and mucinous carcinoma of the ovary. Arch Gynecol Obstet 248: 139-143, 1991.

5. Khurana KK, Tornos C and Silva EG: Ovarian neuroendocrine carcinoma associated with a mucinous neoplasm. Arch Pathol Lab Med 118: 1032-1034, 1994.

6. Jones K, Diaz JA and Donner LR: Neuroendocrine carcinoma arising in an ovarian mucinous cystadenoma. Int J Gynecol Pathol 15: 167-170, 1996.

7. Eichhorn JH, Lawrence WD, Young RH, et al: Ovarian neuroendocrine carcinomas of non-small-cell type associated with surface epithelial adenocarcinomas. A study of five cases and review of the literature. Int J Gynecol Pathol 15: 303-314, 1996.

8. Chen KT: Composite large-cell neuroendocrine carcinoma and surface epithelial-stromal neoplasm of the ovary. Int J Surg Pathol 8: 169-174, 2008.

9. Ohira S, Itoh K, Shiozawa T, et al: Ovarian non-small cell neuroendocrine carcinoma with paraneoplastic parathyroid hormone-related hypercalcemia. Int J Gynecol Pathol 23: 393-397, 2004.

10. Hirasawa T: Ovarian neuroendocrine carcinoma associated with mucinous carcinoma and teratoma. Nippon Rinsho 62: 973-978, 2004.

11. Behnam K, Kabus D and Behnam M: Primary ovarian undifferentiated non-small cell carcinoma, neuroendocrine type. Gynecol Oncol 92: 372-375, 2004.

12. Ahmed Z, Aftab K and Kayani N: Ovarian primary neuroendocrine carcinoma of non-small cell type: report of an extremely rare neoplasm. J Pak Med Assoc 55: 82-84, 2005.

13. Choi YD, Lee JS, Choi C, et al: Ovarian neuroendocrine carcinoma, non-small cell type, associated with serous carcinoma. Gynecol Oncol 104: 747-752, 2007.

14. Lindboe CF: Large cell neuroendocrine carcinoma of the ovary. APMIS 115: 169-176, 2007.

15. Veras E, Deavers MT, Silva EG, et al: Ovarian nonsmall cell neuroendocrine carcinoma: a clinicopathologic and immunohistochemical study of 11 cases. Am J Surg Pathol 31: 774-782, 2007.

16. Dundr P, Fischerová D, Povýsil C, et al: Primary pure large-cell neuroendocrine carcinoma of the ovary. Pathol Res Pract 204: 133-137, 2008

17. Draganova-Tacheva RA, Khurana JS, Huang Y, et al: Large cell neuroendocrine carcinoma of the ovary associated with serous carcinoma with mucin production: a case report and literature review. Int J Clin Exp Pathol 2: 304-309, 2009.

18. NCCN Clinical Practice Guidelines in Oncology (NCCN guidelines TM for Non-Small Cell Lung Cancer) (http://www.ncen. org/professionals/physician_gls/pdf/nscl.pdf). 